\title{
Acceleration of particles to high energy via gravitational repulsion in the Schwarzschild field
}

\author{
Charles H. McGruder III* \\ Department of Physics and Astronomy, Western Kentucky University, Bowling Green, KY 42101
}

(Dated: September 17, 2018)

Gravitational repulsion is an inherent aspect of the Schwarzschild solution of the Einstein-Hilbert field equations of general relativity. We show that this circumstance means that it is possible to gravitationally accelerate particles to the highest cosmic ray energies.

* E-mail me at: mcgruder@wku.edu Visit: ; 


\section{INTRODUCTION}

It is widely believed that there are only two sources of energy available to accelerate cosmic particles to relativistic velocities - magnetic field energy, which accelerates through magnetic connection and kinetic energy, which accelerates through Fermi acceleration (see Drury [1] for a review). However, the recent discovery of pulsed TeV photons from the Crab pulsar contradicts current models of relativistic cosmic particle formation (Ansoldi et al. [2]). Here we point out that there is a third energy source - gravitational energy, which is capable of accelerating particles to the highest cosmic ray energies observed $\left(\sim 10^{20} \mathrm{eV}\right)$.

It is well known that special relativity leads to space, time and mass dependency on velocity. It is however, not so well known, that Einstein's theory of gravitation, general relativity, leads to the dependency of the gravitational acceleration on velocity. In fact, in the Schwarzschild field particles can experience gravitational repulsion as was discovered independently by Droste [3], Hilbert [4, 5]] and Bauer [6]. After decades of debate the existence of gravitational repulsion and the circumstances under which it occurs, was clarified by Treder and Fritze [7] and McGruder [8]. In particular McGruder pointed out that gravitational repulsion can only be detected by distant observers and not by observers located in the Schwarzschild field.

We will show that gravitational repulsion leads to the acceleration of particles to high energy. Although the concept of repulsive gravity associated with gravitating bodies may seem strange to some, a number of authors have employed this concept [9-32].

\section{ACCELERATION AND VELOCITY IN THE SCHWARZSCHILD FIELD}

Einstein [33] and independently Hilbert [34] developed field equations for general relativity (See Janssen \& Renn [35] for details). The spherically symmetric solution of the Einstein-Hilbert field equations for the empty space surrounding a non-rotating point mass was discovered by Schwarzschild [36] and independently by Droste [13]:

$$
d s^{2}=\frac{d r^{2}}{1-\frac{\alpha}{r}}+r^{2}\left(d \theta^{2}+\sin (\theta)^{2} d \phi^{2}\right)-\left(1-\frac{\alpha}{r}\right) d t
$$

$d s$ is the line element, $t$ Schwarzschild time coordinate, $r$ Schwarzschild radial coordinate, $\theta$ colatitude, $\phi$ longitude and $\alpha$ is the Schwarzschild radius, which is:

$$
\alpha=2 G M
$$

where $M$ is the mass of the gravitating body and $c$ the speed of light is 1 . When $r \rightarrow \infty$ equation (1) becomes the Minkowski metric of special relativity. It is important to understand that the Schwarzschild coordinates in equation (1), as well as the Schwarzschild velocities and accelerations are quantities measured by a distant observer, who is not located in the gravitational field of the point mass. Thus, they are the quantities an astronomer perceives.

For purely radial motion the Schwarzschild gravitational acceleration, $\frac{d^{2} r}{d t^{2}}$, experienced by a particle in the Schwarzschild field is (McGruder [8]):

$$
\frac{d^{2} r}{d t^{2}}=g\left[\frac{3}{1-\frac{\alpha}{r}}\left(\frac{d r}{d t}\right)^{2}-\left(1-\frac{\alpha}{r}\right)\right]
$$

where

$$
g=\frac{G M}{r^{2}}
$$

We see that it depends upon the Schwarzschild velocity, $\frac{d r}{d t}$. Therefore, to compute the gravitational acceleration we require an expression for the velocity. It was first derived by Droste [3] and independently by Hilbert [4, 5] and confirmed by Treder and Fritze [7]:

$$
\left(\frac{d r}{d t}\right)^{2}=\left(1-\frac{\alpha}{r}\right)^{2}+A\left(1-\frac{\alpha}{r}\right)^{3}
$$

where $\mathrm{A}$ is a constant. Differentiation of equation (5) yields (3). Equation (5) reduces for $r \rightarrow \infty$ to:

$$
v^{2}=\left(\frac{d r}{d t}\right)^{2}=1+A
$$

where $v$ is the velocity according to special relativity. If $v=0$, then equation (6) leads to $A=-1$. This refers to free fall motion from infinity. It is discussed in detail by Treder and Fritze [7]. 


\section{ACCELERATION OF PARTICLES TO HIGH ENERGY VIA GRAVITATIONAL REPULSION}

From Droste [3] and Hilbert [4, 5] and confirmed by Treder and Fritze [7] and McGruder [8], we learn that gravitational repulsion occurs when:

$$
\frac{d r}{d t}>\frac{1}{\sqrt{3}}\left(1-\frac{\alpha}{r}\right)
$$

Inequality (7) tells us that all particles in the Schwarzschild field with $\frac{d r}{d t}>\frac{1}{\sqrt{3}}$ experience gravitational repulsion. Figure 1 shows the areas of gravitational attraction and repulsion. If the Schwarzschild field contains only a single particle, which is moving radially outward and experiencing gravitational repulsion it will continue to do so until $r \rightarrow \infty$. B. Wieb VanDerMeer (private communication) has pointed out that this idea is apparently not new because Droste [3] states (written in Dutch and translated by VanDerMeer): “.... the acceleration never becomes zero; in that case there is according to equation (54) repulsion and the velocity is maximal at infinite distance; during the motion there is always repulsion".

As noted above when $r \rightarrow \infty$ we have the Minkowski metric of special relativity. The special relativistic kinetic energy is:

$$
E=(\gamma-1) m c^{2}
$$

where $\gamma$ is the Lorentz factor given by:

$$
\gamma=\frac{1}{\sqrt{1-v^{2}}}
$$

Inserting (6) into this expression we obtain:

$$
A=-\frac{1}{\gamma^{2}}
$$

As an example if we let $E=10^{20} \mathrm{eV}$ in equation (8), which is approximately the highest energy cosmic rays observed and insert the proton rest mass in (8), we obtain: $\gamma=1.06579 \times 10^{11}$. Plugging $\gamma$ into equation (10) leads to: $A=-8.80354 \times 10^{-23}$. If we consider a single particle which is moving radially outward and obeys inequality (7), then it will experience gravitational repulsion to $r \rightarrow \infty$, ending up with $E=10^{20} \mathrm{eV}$. Figure 2 depicts the Schwarzschild velocity according to equation (5) out to $r=10 \alpha$. Our numbers are only an example and it is certainly possible to achieve even higher particle energies.

We now turn to the gravitational acceleration in the Schwarzschild field, which is given by equation (3). Figure 3 is a plot of equation (3) out to $r=10 \alpha$. It shows that for our example the gravitational acceleration is always positive, meaning that gravitational repulsion is taking place. The figure also shows that as the distance becomes large, the acceleration approaches $+2 \mathrm{~g}$ (McGruder [8]), where g is given by equation (4).

Finally, we note that unlike electrostatic and magnetic forces, which require charged particles, the gravitational acceleration of elementary particles is only slightly charge dependent as McGruder [37] has shown. This means that uncharged particles like neutrons and neutrinos can be accelerated to very high energies too.

\section{ACKNOWLEDGMENTS}

Many thanks to Dr. and Mrs. William McCormick, whose generous support has provided the prerequisite financial basis and most importantly the necessary time to complete this project.

[1] L. Drury, Origin of Cosmic Rays, Astro. Part. Phys. 39, 52 (2012)

[2] S. Ansoldi, et al., Teraelectronvolt pulsed emission from the Crab Pulsar detected by MAGIC, Astron. Astrophys. 585, A133 (2016)

[3] J. Droste, Het zwaartekrachtsveld van een of meer lichamen volgens de theorie van Einstein, (E. J. Brill, Leiden, 1916)

[4] D. Hilbert, Die Grundlagen der Physik (zweite Mitteilung), Nachrichten von der Gesellschaft der Wissenschaften zu Göttingen, Mathematisch-Physikalische Klasse 53 (1917)

[5] D. Hilbert, Die Grundlagen der Physik, Math. Ann. 92, 1 (1924) 
[6] H. Bauer, Mathematische Einführung in die Gravitationstheorie Einsteins, Leipzig (1922)

[7] H. Treder and K. Fritze, Die Fallbeschleunigung in der Schwarzschild-Metrik, Astron. Nachr. 296, 109 (1975)

[8] C. McGruder, Gravitational Repulsion in the Schwarzschild field, Phys. Rev. D, 25, 3195 (1982)

[9] N. Gorkavyi and A. Vasilkov, A repulsive force in the Einstein theory, MNRAS 461, 2929 (2016)

[10] N. Santos and V. Satheeshkumar, Some Surprises in Relativistic Gravity, arXiv:1606.08300 (2016)

[11] A. Spallicci and M. van Putten, Gauge dependence and self-force in Galilean to Einsteinian free fall, Pisa tower at the general relativity centennial, Int. J. Geom. Meth. Mod. Phys. 13, 1630014, special volume in Memory of Mauro Francaviglia. arXiv:1607.02594 [gr-qc] (2016)

[12] A. Spallicci and P. Ritter, A fully relativistic radial fall, Inter. J. Geom. Meth. Mod. Phys. 11, id 1450090. arXiv:1407.5391 [gr-qc] (2014)

[13] J. Droste, Field of a single center in Einstein's theory of gravitation, and the motion of a particle in that field, Kon. Ak. Wetensch. Proc. 19, 197 (1917)

[14] M . Kutschera and W. Zajiczek, Shapiro effect for relativistic particles - testing General Relativity in a new window, arXiv:0906.5088 (2009)

[15] F.Winkler, The Geodesic Equations and the Mass-Energy Relation from the Coordinate Perspective, Int. J. Theor. Phys. 52, $3045(2013)$

[16] M. Katanaev, Point massive particle in General Relativity, Gen. Relativ. Gravit. 45, 1861 (2013)

[17] A. Spallicci, Free fall and self-force: an historical perspective, in Mass and Motion in General Relativity, Springer Series on Fundamental Theories of Physics, L. Blanchet, A. Spallicci, B. Whiting Eds, ISBN: 978-90-481-3014-6. arXiv:1005.0611 physics.hist-ph] (2011)

[18] M. Barua, Gravitational repulsion by a Reissner-Nordström Black Hole, Ind. J. Pure App. Phys. 23, 634 (1985)

[19] J. Gariel, N. Santos, J. Silk, 2014, Phys. Rev. D90, id.063505

[20] A. Loinger and T. Marsico, On Hilbert's Gravitational Repulsion (A Historical Note), arXiv:0904.1578 (2009)

[21] J. Gariel, N. Santos, J., A. Wang, 2016, Gen. Relativ. Gravit. 48, id. \#66

[22] J. Broekaert, A "Lorentz-Poincare" - Type Interpretation of Relativistic Gravitation, in "Beyond Einstein - Physics for the 21st Century" (2006)

[23] J. Broekaert, A spatially-VSL gravity model with 1-PN limit of GRT, Found. Phys. 38, 409 (2008)

[24] L. Herrera and N. Santos, A General Relativistic Effect in quasi-spherical Objects as the possible Origin of Relativistic Jets, Astrophys. Space Sci. 310, 251 (2007)

[25] F. Felber, Exact 'antigravity-field' solutions of Einstein's equation, arXiv:0803.2864 (2008)

[26] C. Chicone and B. Mashhoon, Explicit Fermi Coordinates and Tidal Dynamics in de Sitter and Gödel Spacetimes, Phys. Rev. D 74, id. 064019 (2006)

[27] A. Loinger and T. Marsico, Protons do not exert any Hilbertian gravitational repulsion, arXiv:0912.1323 (2009)

[28] L. Herrera, Geodesics in a Quasi-Spherical Spacetime - A case of Gravitational Repulsion, Found. Phys. Lett. 18, 21 (2005)

[29] I. Denisova, B. Mehta and A. Zubrilo, The Investigation of the Model of Gravitational Repulsion in Einstein's General Theory of Relativity, Gen. Relativ. Gravit. 31, 821 (1999)

[30] J. Ponce de Leon, Gravitational repulsion in sources of the Reissner-Nordström field, J. Math. Phys. 29, 197 (1988)

[31] K. Krori and M. Barua, Gravitational repulsion by Kerr and Kerr-Newman black holes, Phys. Rev. D, 31, 3135 (1985)

[32] K. Krori, J. Sarmah and D. Goswami, Gravitational repulsion in the Einstein-zero-mass scalar theory, Can. J. Phys. 62, $629(1984)$

[33] A. Einstein, Die Feldgleichungen der Gravitation, Sitzungsberichte der Königlich Preussischen Akademie der Wissenschaften (Berlin) 884 (1915)

[34] D. Hilbert, Die Grundlagen der Physik (Erste Mitteilung), Nachrichten von der Gesellschaft der Wissenschaften zu Göttingen, Mathematisch-Physikalische Klasse 395 (1915)

[35] M. Janssen and J. Renn, Phy. Today 68, 30 (2015)

[36] K. Schwarzschild, Über das Gravitationsfeld eines Massenpunktes nach der Einsteinschen Theorie, Sitzungsberichte der Königlich Preussischen Akademie der Wissenschaften zu Berlin, 189 (1916)

[37] C. McGruder, Field energies and principles of equivalence, Nature 272, 806 (1978) 